\title{
In vivo long-term continuous observation of gene expression in zebrafish embryo nerve systems by using harmonic generation microscopy and morphant technology
}

\author{
Cho-Shuen Hsieh \\ National Taiwan University \\ Department of Electrical Engineering \\ and \\ Graduate Institute of Photonics and Optoelectronics \\ Taipei 10617, Taiwan
}

\section{Ching-Yi Ko}

National Taiwan Ocean University Institute of Bioscience and Biotechnology Keelung 20224, Taiwan

\section{Szu-Yu Chen}

Tzu-Ming Liu

National Taiwan University

Department of Electrical Engineering and

Graduate Institute of Photonics and Optoelectronics Taipei 10617, Taiwan

\author{
Jian-Shiung Wu \\ Chin-Hwa Hu \\ National Taiwan Ocean University \\ Institute of Bioscience and Biotechnology \\ Keelung 20224, Taiwan
}

\section{Chi-Kuang Sun}

National Taiwan University

Department of Electrical Engineering and

Graduate Institute of Photonics and Optoelectronics Academia Sinica

Research Center for Applied Sciences

Taipei 10617, Taiwan

\begin{abstract}
Gene expression plays an important role in embryo development and organ function. Previous studies have shown that harmonic generation microscopy (HGM) can be used as a fluorescence signal-independent, minimally invasive method with a subcellular 3-D resolution and a penetration depth in the order of millimeters for long-term continuous imaging of vertebrate embryos. We show that it is ideal to combine in vivo HGM with the morphant technology for minimally invasive, long-term continuous observation of gene expression in the nervous system of vertebrate embryos. Since second- and third-harmonic generations (SHG, THG) are virtual-state-transitionbased systems that depend only on the structure of the organisms, they are not temporally limited by the expression of the fluorescence proteins. We successfully identified the expression of the zarnt2a and the hif- $1 \alpha, 2 \alpha$, and $3 \alpha$ genes in the nervous system of zebrafish embryos with specific knockdown genes by microscopically observing the embryos from the early stages of embryogenesis. The results from a combination of the two different modalities, i.e., SHG microscopy and THG microscopy, successfully revealed the weak cell adhesion, cell apoptosis, nerve formation reduction, and neural tube distortion in the morphant zebrafish embryos. ๑ 2008 Society of Photo-Optical Instrumentation Engineers. [DOI: 10.1117/1.3050423]
\end{abstract}

Keywords: in vivo imaging; harmonic generation microscopy; third harmonic generation; second harmonic generation; three-photon excited fluorescence; embryology; gene expression; morphant technology.

Paper 08063RR received Feb. 21, 2008; revised manuscript received Nov. 3, 2008; accepted for publication Nov. 4, 2008; published online Dec. 30, 2008.

\section{Introduction}

Genes are the chemical, physical, and functional units of heredity. Genes, which are made up of DNA, act as instructors in the production of proteins. Gene expression plays an important role in embryo development and organ function. The vertebrate genome contains about 50,000-100,000 genes, many of which have unknown functions. In this study, we focused on the continuous long-term in vivo observation of gene expression in the nervous system. Because the nervous

Address all correspondence to Chi-Kuang Sun, National Taiwan University, Department of Electrical Engineering and Graduate Institute of Photonics and Optoelectronics, Taipei 10617, Taiwan; Tel: +866-2-33665085: Fax: +866-2 33663614; E-mail: sun@cc.ee.ntu.edu.tw; Chin-Hwu Hu, National Taiwan Ocean University, Institute of Bioscience and Biotechnology, Keelung 20224 Taiwan; Tel: +866-2-24622192 ext. 5506; Fax: +866-2-24622320; E-mail: chhu@mail.ntou.edu.tw system has a significant influence on sensory reactions, body actions, and organ function, we decided to investigate the genes involved in nerve development.

Many live imaging systems are used to study gene expression in neurology. Magnetic resonance imaging (MRI) determines which parts of the brain are activated by different types of physical sensation or activity, such as the movement of a subject's fingers, visual stimulus, and auditory stimulus. MRI is also used to detect the overexpression of genes in the brain, ${ }^{1}$ but this technique needs sensitive magnetic resonance contrast agents. ${ }^{2}$ Ultrasound can be used for determining the developmental effects of targeted genetic or cellular interventions at critical stages of placentation, gastrulation, axis formation, and neural tube closure. ${ }^{3}$ However, this technique

1083-3668/2008/13(6)/064041/7/\$25.00 @ 2008 SPIE 
cannot reveal neural activity down to the cellular level. Confocal fluorescence ${ }^{4}$ and multiphoton fluorescence microscopy ${ }^{5}$ can study some in vivo changes in gene expression during embryo development, but these techniques are limited by the binding and expression time of fluorescence proteins. ${ }^{6}$ Harmonic generation microscopy (HGM), which is known to leave no energy deposition on the interacted matter due to HGM's energy conservation characteristic, ${ }^{7}$ supports the least optically invasive nature desirable for in vivo long-term continuous observation in studying developmental processes. Similar to confocal and multiphoton microscopy, HGM is used with subcellular-level 3-D resolution, while the technique's millimeter penetration capability ${ }^{8}$ makes HGM an excellent tool for studying gene expression during embryogenesis. Without the aid of fluorescence protein, nerve fibers ${ }^{9}$ and spindle fibers ${ }^{10}$ can be directly revealed through the secondharmonic generation (SHG) signals, and cell membranes ${ }^{8}$ can be directly revealed through the third-harmonic generation (THG) signals, making HGM an ideal tool for studying nerve fiber formation, cell division activity, and organ morphology related to different gene functions.

The morphant technique is straightforward to perform, competent for action in all cell types, efficient at depleting the selected protein, and amenable to the targeting of many genes. For gene function studies, it can act as an in vivo gene targeting tool. ${ }^{11}$ Morpholino phosphorodiamidate oligonucleotides [morpholinos (MOs)] are synthetic DNAs that contain a MD ring and a neutral charge backbone and have high affinity to ribonucleic acids (RNAs). ${ }^{11}$ By binding to and blocking the translation of mRNAs, MOs have been successfully used in a variety of systems for gene knockdown studies. The zebrafish, being a standardized vertebrate model, is viewed as an appropriate animal model for morphant technology. Zebrafishes have definite developing stages, their genome sequence has been completely discovered, and much of their genetic material is the same as that of humans. The zebrafish's rapid rate of development, precisely defined developing stages, transparency, small size, and external development capability facilitate many genomic studies and microscopic observations. ${ }^{12}$ Formal optical techniques used in morphant technology research, such as staining, biopsy, and optical microscopy, cannot in vivo trace the gene expression in the same living zebrafish for an extended period. Optical higher-HGM can continuously observe zebrafish embryos, including cell morphology of the nervous system, for over $20 \mathrm{~h}$ with no photodamage effects, ${ }^{12}$ making it ideal for morphant technology research.

In this paper, we report the application of HGM for continuous observations during in vivo morphant zebrafish embryo studies. We focused our studies on the genes zarnt $2 a$ (aryl hydrocarbon receptor nuclear translocator) and hif (hypoxia inducible factor)- $1 \alpha, 2 \alpha, 3 \alpha$ because of their influence on the nervous system from the very beginning of embryo development. Weak cell adhesion during the gastrula period and cell apoptosis during the pharyngula period were revealed through THG modality. With SHG modality, the decrease in nerve fiber formation in the forebrain of the embryos due to the blocking of these genes could be observed. Our study indicates that combining in vivo continuous HGM imaging with morphant technologies can offer valuable insight into gene functions in vertebrate embryo development, especially in the early developing stage, complementary to other existing technologies.

\section{Materials and Methods}

Our laser-scanning harmonic generation microscope ${ }^{12}$ was adapted with an Olympus FV300 scanning unit along with an Olympus BX51 microscope. The collimated Cr:forsterite laser beam, whose center wavelength was $1230 \mathrm{~nm}$ with a repetition rate of $110 \mathrm{MHz}$ and a pulse width of $140 \mathrm{fs}$, was coupled into the scanning system as the excitation source. To observe the whole 3-D structure of the zebrafish brain, we used a 2-mm-working-distance, high-numerical-aperture (NA) infrared objective (LUMPlanF1/IR 60X/water/NA0.9, Olympus) to focus the laser beam on the desired location of the specimen with a spot size close to its diffraction limit. The average power after the objective was approximately $150 \mathrm{~mW}$. Taking advantage of the transparence and finite thickness of the live specimen, we used a NA0.9 air condenser to collect higher harmonic generation signals in the forward direction. The SHG and THG signals were separated by a beam splitter and were guided into two photomultiplier tubes (PMTs) to record the filtered SHG and THG signals, respectively. For the THG signal origin study, filtered threephoton fluorescence with a center wavelength at $535 \mathrm{~nm}$ was collected using the focusing infrared objective in the epidirection for PMT detection.

The zarnt $2 a$-specific morpholino-modified-antisenseoligonucleotides (MOs) were designed to target the zebrafish ARNT2A (GenBank AF055166) (5'-GGTTTACAGCGGCTGGTGTTGCCAT-3') cDNA (nt 221-245). ${ }^{13}$ The hif- $1 \alpha$-specific MOs were designed to target the zebrafish hif-1 $\alpha$ (GenBank AY326951) (5'CAGTGACAACTCCAGTATCCATTCC-3') cDNA (nt 235259). The hif- $2 \alpha$-specific MOs were designed to target the zebrafish HIF-2 $\alpha$ (GenBank DQ375242) (5'CGCTGTTCTCGCGTAATTCCCGCAG-3') cDNA (nt 223247). The hif-3 $\alpha$-specific MOs were designed to target the zebrafish HIF-3 $\alpha$ (GenBank AY330295) (5'CCTTTTCGACGTAGAGTTCACCATC-3') cDNA (nt 92116). The four MOs were obtained from a commercial supplier (Gene Tool, Philomath, OR $)^{13}$ and were microinjected into the zebrafish embryos. ${ }^{14}$ The dosage of MOs represents the total weight of MOs injected into one embryo. For example, a dose of 12-ng zarnt2a means that 12-ng zarnt $2 a$ MOs were injected, but a mixed dose of 18-ng hif- $1 \alpha, 2 \alpha$, $3 \alpha$ means that 6-ng hif-1 $\alpha$ MOs, 6-ng hif-2 $\alpha$ MOs, and 6-ng hif-3 $\alpha$ MOs were injected simultaneously into one embryo. These genes have great influence on the nervous system, ${ }^{13}$ including the forebrain, midbrain, and somite. In a preliminary study we observed that in separate hif-1 $\alpha$, hif- $2 \alpha$, or hif- $3 \alpha$ morphant zebrafish embryos, the blocking of the hypoxia response was not full, probably because hif- $1 \alpha$, hif- $2 \alpha$, and hif- $3 \alpha$ MOs have similar functions, so they have a genetic redundancy problem. To overcome this problem, we completely blocked the hypoxia response by injecting hif- $1 \alpha$, hif- $2 \alpha$, and hif-3 $\alpha$ MOs simultaneously. Because of the ease of identification and observation, we focused on the development of the forebrain. For each embryo, we performed continuous HGM observation for over $17 \mathrm{~h}$, and observation of different morphant zebrafish embryos was 

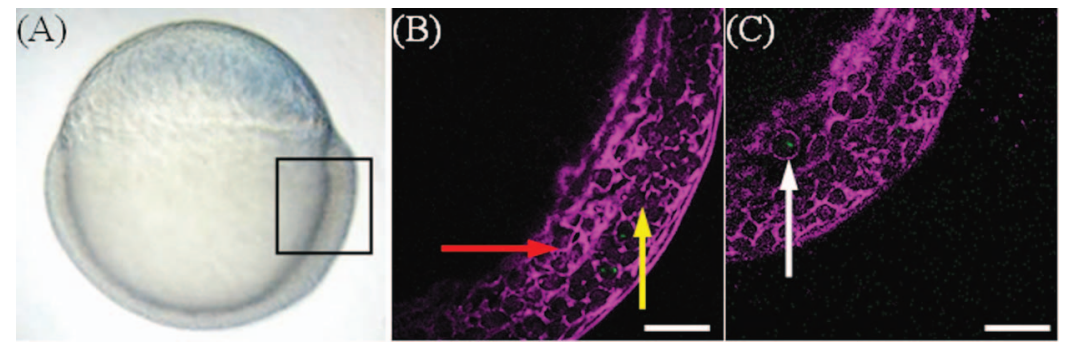

Fig. 1 THG (shown in purple) sectioned in vivo microscopic images of (b) control and (c) zarnt2a morphant zebrafish embryos at 7 hpf. During epiboly, the boundary between ectoderm (yellow arrow) and mesoderm (red arrow) can be distinguished by the THG signals. The white arrow indicates the position of the isolated cell. The black square in (a) indicates the imaging area. Scale bar: $50 \mu \mathrm{m}$.

repeated more than 10 times with different embryos. Although there were variations between morphants of a single type, the tendencies of decreasing nerve fiber and neural tube distortion in the forebrain, as discussed below, were much the same in all embryos. Therefore, only one of the typical results representing different morphant zebrafish is shown in the following discussion. Whole-mount in situ hybridization was also performed as previously described. ${ }^{15}$ To make gene-specific probes, digoxigenin-labeled cRNA fragments containing 1069 bp (nt 1800-2850) of the R-cadherin gene (GenBank $\mathrm{BC} 116496)$ and $500 \mathrm{bp}$ (nt 55-554) of the HuC gene (GenBank U62018) were synthesized with the DIG RNA Labeling Kit (Sp6/T7, Roche, USA). Hybridization was detected by anti-digoxigenin (DIG) antibody coupled to alkaline phosphatase. For controls, a random sequence (RS) morpholino (the standard control morpholino) available from Gene Tools was used as an injection control. For the zarnt $2 a$ morphant zebrafish embryo experiments, a dose of 12-ng random sequence MO was injected into the embryos as a control, while in the hif-1 $\alpha, 2 \alpha, 3 \alpha$ morphant zebrafish embryo experiments, a dose of 18-ng random sequence MO was injected into the embryos as controls.

\section{Results and Discussions}

Because of the Gouy phase shift effect in a tightly focused beam, THG occurs when the focus is located at the interface of two media with different linear or nonlinear optical indices. ${ }^{16}$ Because SHG can arise from highly organized nanostructures with an ordered arrangement of asymmetric biomolecules where the optical centrosymmetry is broken, ${ }^{17}$ SHG can reveal spindles and nerve fibers. ${ }^{9,10}$ Northern hybridization analysis study and in situ hybridization showed that zARNT2A mRNA was transcribed in the brain and has great influence on the nervous system. ${ }^{13}$ However, it is not known how zARNT2A mRNA influences nerve development. Therefore, the zarnt $2 a$ morphant zebrafish serves as a suitable animal model to test if continuous HGM observation can be combined with morphant technology for gene expression studies. At the very beginning of zebrafish embryo development, we observed a conspicuous diversity between the control and the zarnt $2 a$ morphant zebrafish embryos. Figure 1 shows the THG-sectioned in vivo microscopic images from the two types of embryos at 7-h postfertilization (hpf). Epiboly, which begins in the late blastula period, causes the thin-

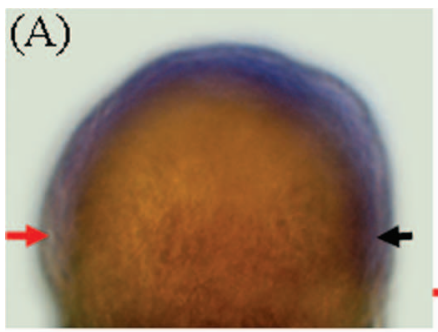

(E)

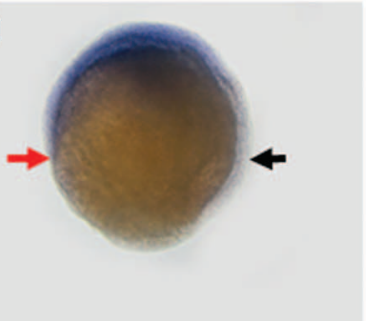

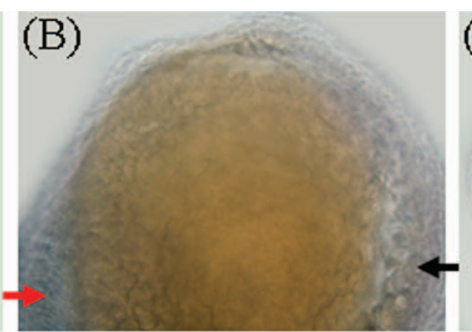

(C)

(F)

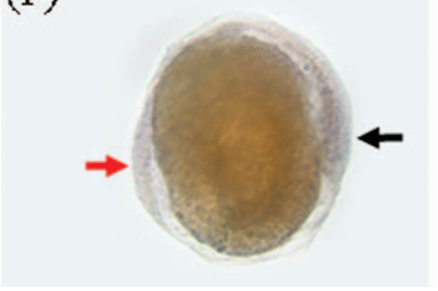

\section{(C)}

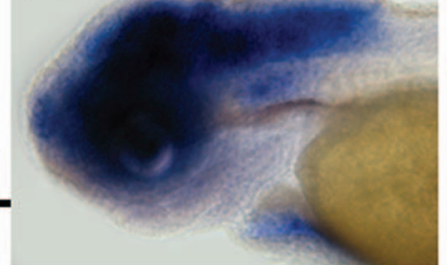

(G)

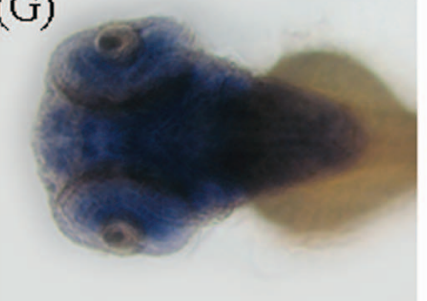

(D)

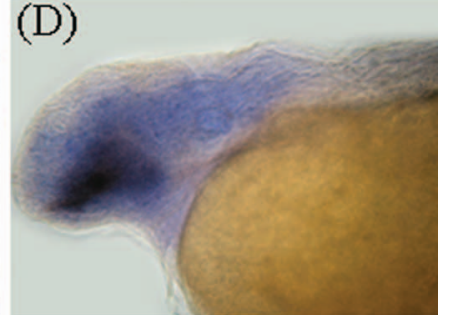

(H)

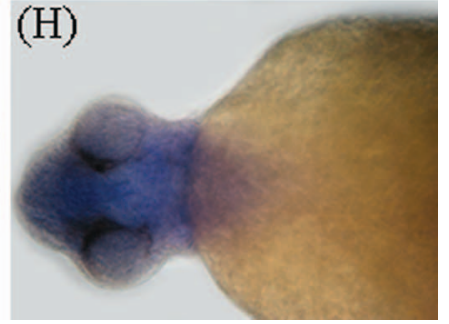

Fig. 2 The blocking of the zarnt2a gene causes weak cell-cell adhesion in zebrafish embryos at $7 \mathrm{hpf}(\mathrm{a}),(\mathrm{b}),(\mathrm{e}),(\mathrm{f})$ and $72 \mathrm{hpf}(\mathrm{c}),(\mathrm{d}),(\mathrm{g}),(\mathrm{h})$. $z R$-cadherin (zcdh-4) is expressed in the controls largely (a), (c), (e), (g) but expressed weakly in the zarnt2a morphant zebrafish embryos (b), (d), $(\mathrm{f})$, (h). The top of (a), (b), (e), and (f) is the animal pole of zebrafish embryos. The black arrows show the dorsal region. The red arrows show the ventral region. (c), (d): Lateral view. (g), (h): Dorsal view. 

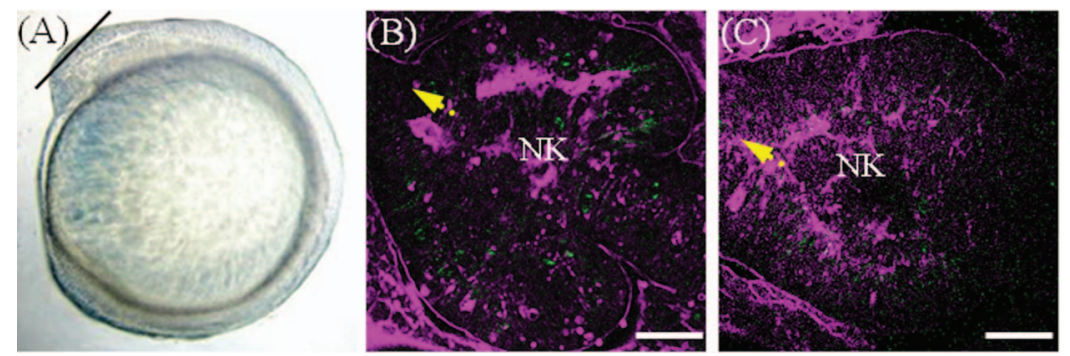

Fig. 3 In vivo HGM images in the neural keel (NK) of (b) control and (c) zarnt2a morphant zebrafish embryos at 12 hpf. The embryos were settled with the dorsal side to the top, and the yellow arrow shows the posterior-to-anterior direction. Compared to the straight nerve fibers revealed by the SHG signals (shown in green) in the control embryos, the SHG signals were much fewer and weaker in the morphant zebrafish embryos. The THG signals are denoted by the purple color. The black line in (a) shows the sectioned region. Scale bar: $50 \mu \mathrm{m}$.

ning and extending of both the yolk syncytial layer (YSL) and the blastodisc over the yolk cells. ${ }^{18}$ The yellow arrow in Fig. 1(b) indicates the position of the ectoderm, and the red arrow indicates the position of the mesoderm formed by the migrating cells. Previous THG studies ${ }^{7,8,10,12,19}$ have already indicated that THG modality can reveal cell membranes. As clearly shown in the THG microscopic images of the zarnt $2 a$ morphant zebrafish embryos, some cells can be found to be isolated [white arrow in Fig. 1(c)] with a round shape during epiboly through THG modality. This is an indication that cell adhesion becomes weak due to the blocking of the zarnt $2 a$ gene.

$\mathrm{R}$-cadherin and $\mathrm{N}$-cadherin are both $\mathrm{Ca}^{2+}$-dependent cellcell adhesion molecules. Moreover, they can form a cisheterodimer to generate new functional units to mediate cellcell adhesion. ${ }^{20,21}$ To further investigate the HGM-observed weak cell adhesion in zarnt $2 a$ morphants, we analyzed the expression of $z R$-cadherin $(z c d h-4)$ of the control and zarnt $2 a$ morphant zebrafish embryos at $7 \mathrm{hpf}$. As shown in Fig. 2, at $7 \mathrm{hpf}$ we observed that $z R$-cadherin $(z c d h-4)$ was expressed widely [Figs. 2(a) and 2(e)] in the control zebrafish embryos but was expressed much less in zarnt $2 a$ morphant zebrafish embryos [Figs. 2(b) and 2(f)]. Even at the 72-hpf stage, the expression of $z R$-cadherin $(z c d h-4)$ in the zarnt $2 a$ morphant [Figs. 2(d) and 2(h)] was still significantly less than the expression in the controlled embryos [Figs. 2(c) and 2(g)]. Our analysis further confirms our frequent HGM observation that the knockdown of the zarnt $2 a$ gene in zebrafish causes defects in cell-cell adhesion.

As the long-term in vivo observation continued, the same embryo developed to the 12-hpf stage (shown in Fig. 3). Compared with the early-grown straight nerve fibers (revealed through SHG modality) in the neural keel of the control set, the SHG signals in the neural keel of the zarnt $2 a$ morphant zebrafish embryos were found to be much fewer and weaker. Our continuous observation revealed that in the control set, in the zebrafish embryos' forebrain, the lumen formed through cavitation, and the SHG signals revealed that numerous nerve fibers had developed in the neural tube at approximately $24 \mathrm{hpf}^{12}$ However, due to the lack of zARNT2A proteins, the SHG signals in the forebrain of the morphant zebrafish revealed the greatly reduced nerve fiber development compared to the controls (Fig. 4). Greatly suppressed nerve fiber development in the zarnt $2 a$ morphant zebrafish's forebrain, indicated by the abatement and weakening of the SHG signals, might be directly or indirectly related to the observed weak cell adhesion revealed by the THG signals during the gastrula period. According to the fate map of zebrafish, the fate of the blastoderm cells appears to be fixed shortly before gastrulation begins. ${ }^{18}$ The cells in the ectoderm will give rise to such tissues as the epidermis, the central nervous system, and the neural crest. The weak cell adhesion during gastrulation caused by the lack of zARNT2A would influence the formation of the three germ layers and could thus lead to the later reduction in nerve fiber development in the forebrain, as indicated by the SHG signals.

Because continuous HGM observation of the zarnt $2 a$ morphant zebrafish had successfully identified some zarnt $2 a$ gene function in the nervous system from the very beginning of embryogenesis, we applied HGM to study the influence of the
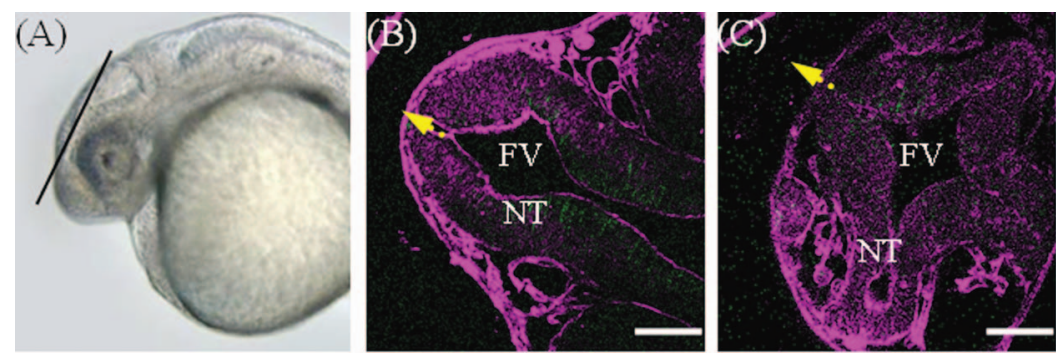

Fig. 4 In vivo HGM images in the forebrain of (b) control and (c) zarnt2a morphant zebrafish embryos at 24 hpf. The yellow arrow shows the posterior-to-anterior direction. At $24 \mathrm{hpf}$ the forebrain ventricle is surrounded by the neural tube. From our HGM observations, in the control zebrafish embryos there were numerous nerve fibers developed in the neural tube perpendicular to the edge of the ventricular zone. The lacking of the ZARNT2A protein influences the formation of nerve fibers in morphant zebrafishs. The SHG and THG signals are denoted by green and purple colors respectively. NT, neural tube; FV, forebrain ventricle. The black line in (a) shows the sectioned region. Scale bar: $50 \mu \mathrm{m}$. 

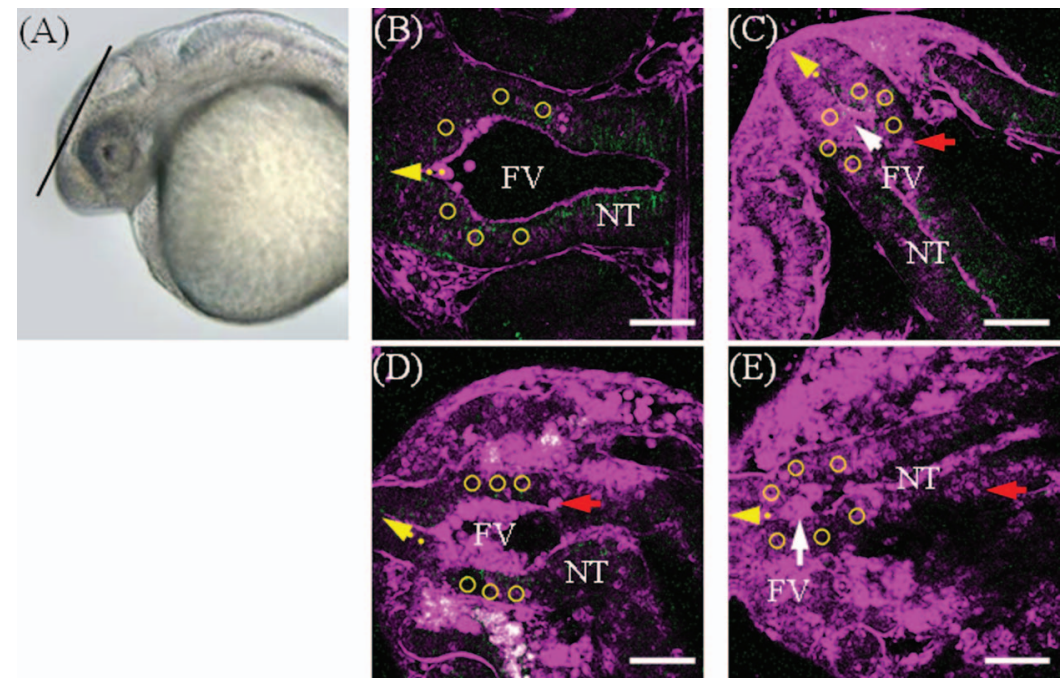

Fig. 5 Dosage dependent in vivo HGM sectioned images of the hif- $1 \alpha, 2 \alpha, 3 \alpha$ morphant zebrafishs' forebrain at 24 hpf. The SHG signals (shown in green) of the straight nerve fibers decrease as the dosage of $\mathrm{MO}$ increases in each zebrafish from (b) control, (c) $9 \mathrm{ng}$, (d) $18 \mathrm{ng}$, to (e) $27 \mathrm{ng}$. Yellow circulars represent the areas selected for quantitative SHG analysis. The THG signals are denoted by the purple color. The yellow arrows show the posterior-to-anterior direction. The red arrows indicate three of the many apoptosis cells. NT, neural tube; FV, forebrain ventricle. The black line in (a) shows the sectioned region. Scale bar: $50 \mu \mathrm{m}$.

unknown hif-1 $\alpha, 2 \alpha, 3 \alpha$ genes, which have partial overlap with the zarnt $2 a$ gene in sequence, on nerve fiber development. Figure 5 shows an example of in vivo HGM-sectioned images of the forebrain of the control zebrafish and the hif-1 $\alpha, 2 \alpha, 3 \alpha$ morphant zebrafish with different applied dosages at 24 hpf. Compared with the control zebrafish [Fig. $5(\mathrm{~b})]$, the development of nerve fibers was found to be considerably diminished, while serious distortion of neural fibers could be observed when the MO dosage increased. This MO dosage-dependent study suggested that hif- $1 \alpha, 2 \alpha, 3 \alpha$ is related to the formation of nerve fibers in the brain.

To identify the function of the hif- $1 \alpha, 2 \alpha, 3 \alpha$ genes in development of the nervous system more specifically, we quantitatively analyzed the SHG signals of the nerve fibers in the forebrain of the hif- $1 \alpha, 2 \alpha, 3 \alpha$ morphant zebrafish. The ventricular zone is approximately $10 \mu \mathrm{m}$ wide from the edge of the neural tube. ${ }^{12}$ In this region, the SHG signals arise from both the spindle fibers of the neural stem cells and the nerve fibers. Therefore, we selected six zones with the same area size in the neural tube outside the forebrain ventricle zone (more than $10 \mu \mathrm{m}$ from the edge of the neural tube) for each dose (Fig. 5). Thus, we made sure that the SHG signals we analyzed all arose from the nerve fibers. With the same PMT voltage of $1100 \mathrm{~V}$, images of $512 \times 512$ pixels, integration time of $13 \mathrm{~s}$, and laser power of $125 \mathrm{~mW}$ after the objective lens, the SHG signal pixels with contrast lower than 20 were considered to be noise. Figure 6 shows the average SHG signal pixels of six zones with contrast above 20 . The figure shows that the SHG signals decrease as the MO dosage increases, and there exists an obvious gap between the control zebrafish embryos (indicated as dosage $0 \mathrm{ng}$ ) and the morphant zebrafish embryos, proving that hif- $1 \alpha, 2 \alpha, 3 \alpha$ is essential for nerve fiber formation.

According to previous studies, ${ }^{22}$ the gene $z H u C$ was expressed in the neuronal precursor cells in the neural plate immediately after gastrulation and then expressed strongly in most regions of the nervous system. ${ }^{22}$ Therefore, $z H u C$, one of the earliest neuronal markers, is an excellent marker gene for observing the developing nervous system in zebrafish embryos. In order to confirm the above result, we examined $z H u C$ expression in the control zebrafish embryos and the hif- $1 \alpha, 2 \alpha, 3 \alpha$ morphant zebrafish embryos of different doses (9 ng, $18 \mathrm{ng}, 27 \mathrm{ng}$ ) at $24 \mathrm{hpf}$. Compared with the control zebrafish embryos, the hif- $1 \alpha, 2 \alpha, 3 \alpha$ morphant zebrafish embryos all revealed minor expression of $z \mathrm{HuC}$ in the forebrain (Fig. 7), which is consistent with the previous result. Hence, these studies suggest that the number of neural cells in the forebrain decreased when the MO dosage increased [Figs. 7(b)-7(d) and 7(f)-7(h)].

In the HGM images we can observe not only the decreasing number of nerve fibers caused by knocking down the hif- $1 \alpha, 2 \alpha, 3 \alpha$ genes, but we can also find widespread apoptosis cells in the forebrains. In the in vivo-sectioned HGM images in the developing forebrain of the hif- $1 \alpha, 2 \alpha, 3 \alpha$ morphants, one can observe many round, solid THG objects

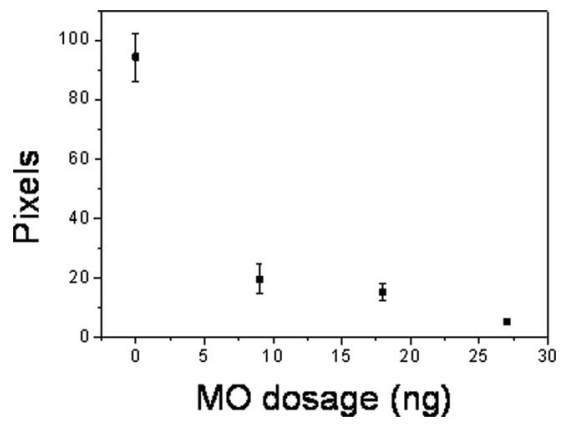

Fig. 6 Pixel numbers of SHG signals with contrast above 20 as a function of $\mathrm{MO}$ dosage in hif- $1 \alpha, 2 \alpha, 3 \alpha$ morphants. The SHG signals decreased as the $\mathrm{MO}$ dosage increased. $0 \mathrm{ng}$ indicates the control data. 


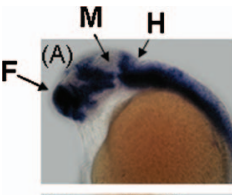

(E) $\mathrm{M} \mathrm{H}$

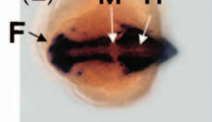

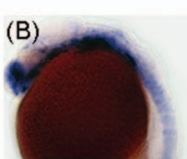

(F)

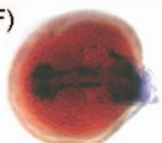

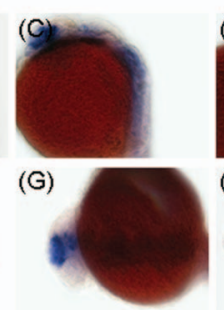

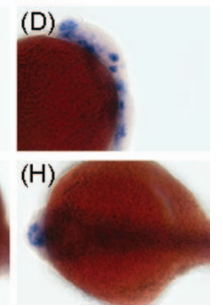

Fig. 7 Expression of $\mathrm{zHuC}$ is less and less with the dosage of $\mathrm{MO}$ increased. Lateral (a), (b), (c), (d), dorsal (e), (f), (g), (h) views of embryos at $24 \mathrm{hpf}$. The expression of $\mathrm{zHuC}$ in the forebrain of control zebrafish (a), (e) is very strong, but the zHuC expression in hif- $1 \alpha, 2 \alpha, 3 \alpha$ [9 ng: (b), (f)], [18 ng: (c), (g)], and [27 ng: (d), (h)] morphant zebrafish embryos is much less when the dosage of $\mathrm{MO}$ increased. $F$, forebrain; $M$, midbrain; $H$, hindbrain.
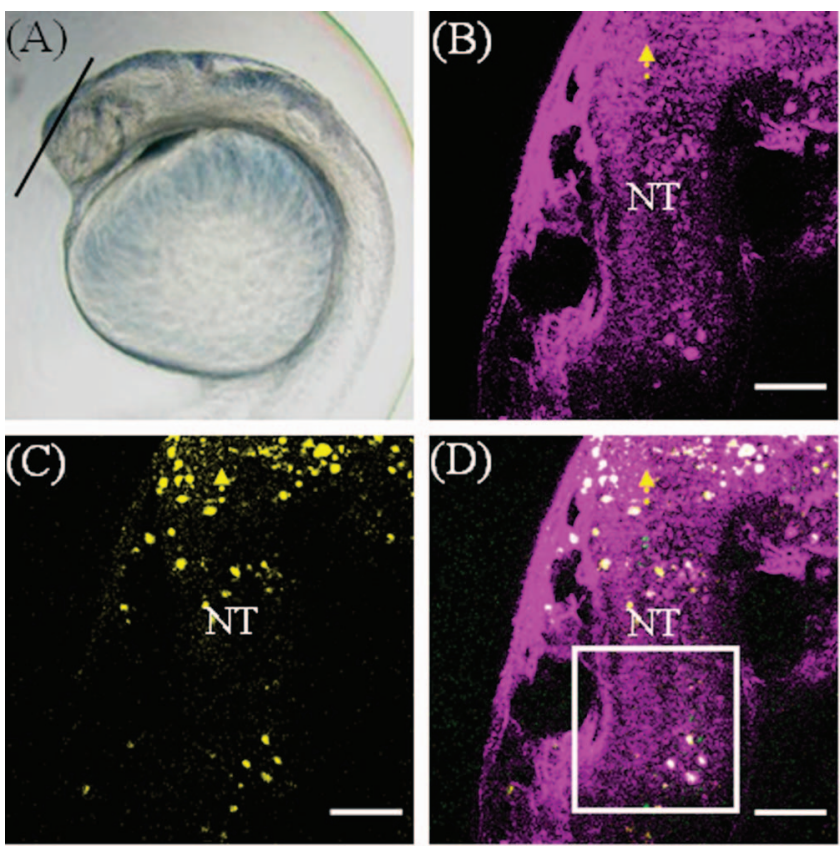

Fig. 8 In vivo (b) THG (shown in purple) and the simultaneously acquired (c) 3PF (shown in yellow) images of the acridine orange stained hif- $1 \alpha, 2 \alpha, 3 \alpha$ morphant zebrafish embryo forebrain at $20 \mathrm{hpf}$. (d) The combined THG/3PF image shows that the round-shaped THC signals were from the apoptotic cells. The yellow arrow shows the posterior-to-anterior direction. NT, neural tube. The black line in (a) shows the sectioned region. Scale bar: $50 \mu \mathrm{m}$.

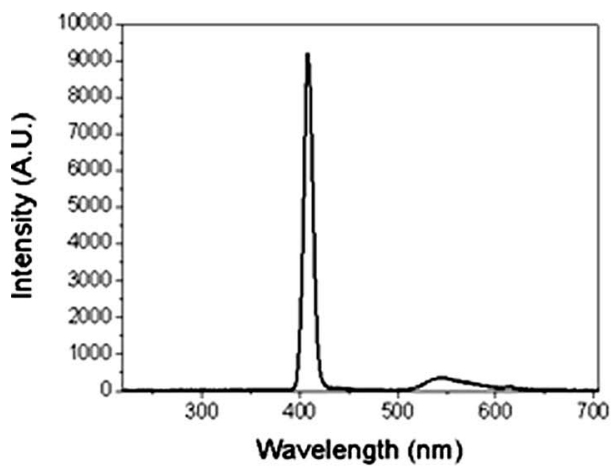

(a) (red arrows in Fig. 5) which were sometimes so dense that they appeared as connected regions. [Please compare the neural tube areas in Fig. 5(b) to Fig. 5(c)-5(e).] To study the source of these round objects reflected through the THG contrast in the neural tubes, we performed many simultaneous staining experiments, and our analysis indicated that the contrast came from apoptosis cells. Figure 8 shows the simultaneously acquired in vivo THG and three-photon-fluorescence (3PF) image of the hif- $1 \alpha, 2 \alpha, 3 \alpha$ morphant zebrafish embryo with acridine orange staining (A6014, Sigma-Aldrich, emission central wavelength $535 \mathrm{~nm}){ }^{23}$ The $3 \mathrm{PF}$ signals from the acridine orange stain labeled the apoptotic cells. The nonlinear emission spectrum of the acridine orange-stained apoptotic cells is given in Fig. 9(a), showing the narrowlinewidth THG at $410 \mathrm{~nm}$ and the broad $3 \mathrm{PF}$ between 525 and $575 \mathrm{~nm}$. The cubic power dependencies of both the 3PF intensity from the acridine orange (centered at $535 \mathrm{~nm}$ ) and the THG intensity (centered at $410 \mathrm{~nm}$ ) are shown in Fig. 9 (b), confirming the three-photon nature of the acridine orange fluorescence. Our 3PF study indicated that the observed solid THG objects are apoptotic cells.

Our study not only presented evidence that apoptotic cells can provide strong THG contrast but also demonstrated strong apoptosis behaviors in the developing forebrain of the studied hif- $1 \alpha, 2 \alpha, 3 \alpha$ morphants. More detailed, long-term in vivo investigations [Fig. 10(b)] revealed abundant mitosis behaviors near the midline of the neural rod through the SHG signals from the mitosis spindles in hif- $1 \alpha, 2 \alpha, 3 \alpha$ morphant zebrafish embryos. Combined with the previous results of strongly decreased nerve fibers, our study indicates that in hif- $1 \alpha, 2 \alpha, 3 \alpha$ morphant zebrafish, the lack of HIF- $1 \alpha, 2 \alpha, 3 \alpha$ proteins does not influence the mitosis of the neural stem cells, which are located in the ventricular zone near the midline of the neural rod, ${ }^{12}$ but influences the formation of nerve fibers. This could be the first in vivo multimode microscopy that includes SHG, THG, and 3PF signals simultaneously.

\section{Summary}

With a deep penetration depth and minimal attenuation in living tissues, a 1230-nm Cr:forsterite laser is ideal for in vivo long-term continuous biological study. Combining the least invasive Cr:forsterite laser-based HGM with well-developed

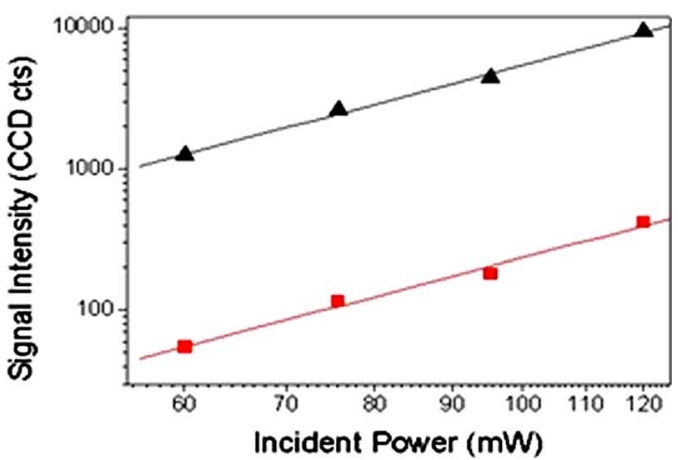

(b)

Fig. 9 (a) Nonlinear emission spectrum from the acridine orange labeled apoptotic cells showing the THG peak at $410 \mathrm{~nm}$ and the broad $3 \mathrm{PF}$ with a center wavelength of $535 \mathrm{~nm}$. (b) Power dependency of the THG signals (triangle) and the 3PF signals (rectangle) of the acridine orange labeled apoptotic cells. The well-matched solid lines are the slope $=3$ fitting, confirming the three-photon nature of both nonlinear processes. 

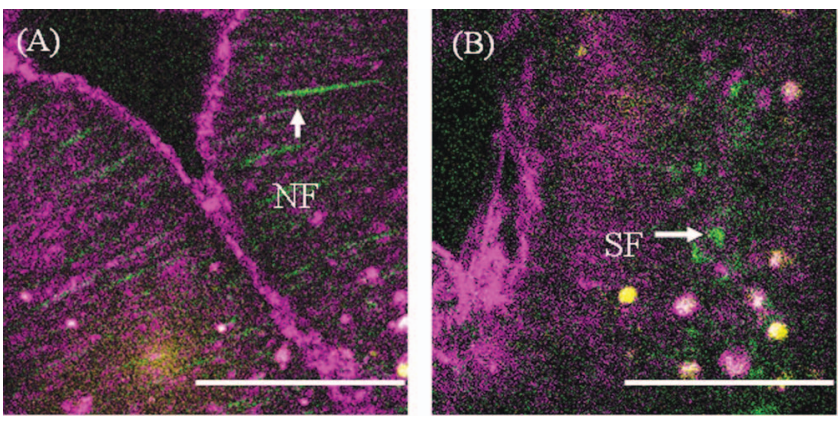

Fig. 10 (a) HGM image of neural tube in control zebrafish forebrain at $20 \mathrm{hpf}$ and (b) enlarged HGM image corresponding to the squared area in Fig. 8(d). The SHG signals from the mitotic spindle fibers can be observed. SHG, THG, and 3PF (acridine orange) signals are denoted by green, purple, and yellow colors, respectively. NF, nerve fiber; SF, spindle fiber. Scale bar: $50 \mu \mathrm{m}$.

morphant technology could reveal the role of different genes without staining. In our in vivo study of the zarnt $2 a$ and hif- $1 \alpha, 2 \alpha, 3 \alpha$ genes in nervous system development, by blocking the expression of these genes, the THG signals revealed weak cell adhesion from the very beginning of embryogenesis, thus affecting the formation of germ layers, which determine the destination of all cells in the embryos. The distortion of the neural tube in the forebrain observed by the THG signals and the decrease in nerve fiber formation observed by the SHG signals indicated that these genes play important roles in the development of the nervous system. In addition to morphology observation, quantitative analysis of the SHG signals in the neural tubes demonstrated the influence of MOs on nerve fiber development through dosagedependent studies. The increased MO dosage led to a quantitative decrease in the number of observed SHG signals in the nerve fibers, and the SHG signal intensity apparently varied between the control and the morphant zebrafish embryos. This proves that blocking the hif- $1 \alpha, 2 \alpha, 3 \alpha$ genes influences the formation of nerve fibers. By using in vivo staining, we have also successfully proven that THG can reveal apoptotic cells. Through long-term continuous observation, our HGM study also suggested that instead of preventing neural stem cells from undergoing mitosis, the lack of hif- $1 \alpha, 2 \alpha, 3 \alpha$ genes affected the formation of nerve fibers. This study shows that combining morphant technology with continuous in vivo HGM observation can provide a new tool for studying gene function in embryology, not only in neurology but from the very beginning of embryogenesis.

\section{Acknowledgments}

This study was sponsored by the National Health Research Institute (NHRI-EX97-9201EI) of Taiwan, the National Science Council (NSC-96-2313-B-019-004; NSC-95-2313-B019-010), Frontier Research of National Taiwan University, and the National Taiwan University Center for Medical Excellence.

\section{References}

1. B. Cohen, K. Ziv, V. Plaks, T. Israely, V. Kalchenko, A. Harmelin, L. E. Benjamin, and M. Neeman, "MRI detection of transcriptional regulation of gene expression in transgenic mice," Nat. Med. 13(4), 498-503 (2004).
2. T. Ichikawa, D. Högemann, Y. Saeki, E. Tyminski, K. Terada, R. Weissleder, E. A. Chiocca, and J. P. Basilion, "MRI of transgene expression: Correlation to therapeutic gene expression," Neoplasia 4(6), 523-530 (2002).

3. J. C. Slevin, L. Byers, M. Gertsenstein, D. Qu, J. Mu, N. Sunn, J. C. Kingdom, J. Rossant, and S. L. Adamson, "High resolution ultrasound-guided microinjection for interventional studies of early embryonic and placental development in vivo in mice," BMC Dev. Biol. 6(10), 1-14 (2006).

4. C. Palmes-Saloma and C. Saloma, "Long-depth imaging of specific gene expressions in whole-mount mouse embryos with single-photon excitation confocal fluorescence microscopy and FISH," J. Struct. Biol. 131(1), 56-66 (2002).

5. E. S. Ruthazer and H. T. Cline, "Multiphoton imaging of neurons in living tissue: Acquisition and analysis of time-lapse morphological data," Real-Time Imag. 8(3), 175-188 (2002).

6. R. M. K. Yu, C. C. Lin, P. K. Chan, E. S. H. Chow, M. B. Murphy, B. P. Chan, F. Müller, U. Strähle, and S. H. Cheng, "Fourdimensional imaging and quantification of gene expression in early developing zebrafish (Danio rerio) embryos," Toxicol. Sci. 90(2), 529-538 (2006).

7. C.-K. Sun, "Higher harmonic generation microscopy," Adv. Biochem. Eng./Biotechnol. 95, 17-56 (2005).

8. S.-W. Chu, S.-Y. Chen, T.-H. Tsai, T.-M. Liu, C.-Y. Lin, H.-J. Tsai, and C.-K. Sun, "In vivo developmental biology study using noninvasive multi-harmonic generation microscopy," Opt. Express 11(23), 3093-3099 (2003).

9. D. A. Dombeck, K. A. Kasischke, H. D. Vishwasrao, M. Ingelsson, B. T. Hyman, and W. W. Webb, "Uniform polarity microtubule assemblies imaged in native brain tissue by second-harmonic generation microscopy," PNAS 100(12), 7081-7086 (2003).

10. C.-K. Sun, S.-W. Chu, S.-Y. Chen, T.-H. Tsai, T.-M. Liu, C.-Y. Lin, and H.-J. Tsai, "Higher harmonic generation microscopy for developmental biology," J. Struct. Biol. 147(1), 19-30 (2004).

11. S. C. Ekker and J. D. Larson, "Morphant technology in model developmental systems," Genesis 30(3), 89-93 (2001).

12. S.-Y. Chen, C.-S. Hsieh, S.-W. Chu, C.-Y. Lin, C.-Y. Ko, Y.-C. Chen, H.-J. Tsai, C.-H. Hu, and C.-K. Sun, "Noninvasive harmonics optical microscopy for long-term observation of embryonic nervous system development in vivo," J. Biomed. Opt. 11(5), 054022 1-8 (2006).

13. W. D. Wang, J. C. Wu, H. J. Hsu, Z. L. Kong, and C. H. Hu, "Overexpression of a zebrafish ARNT2-like factor represses CYP1A transcription in ZLE cells," Mol. Mar Biol. Biotechnol. 2(4), 376-386 (2000).

14. H. J. Hsu, W. D. Wang, and C. H. Hu, "Ectopic expression of negative ARNT2 factor disrupts fish development," Biochem. Biophys. Res. Commun. 282(2), 487-492 (2001).

15. M. Westerfield, "The zebrafish book. A guide for the laboratory use of zebrafish (Danio rerio)," University of Oregon Press, Eugene (2000).

16. T. Y. F. Tsang, "Optical 3rd-harmonic generation at interfaces," Phys. Rev. A 52(5), 4116-4125 (1995).

17. P. J. Campagnola, A. C. Millard, M. Terasaki, P. E. Hoppe, C. J. Malone, and W. A. Mohler, "Three dimensional high-resolution second-harmonic generation imaging of endogenous structural proteins in biological tissues," Biophys. J. 82(1), 493-508 (2002).

18. C. B. Kimmel, W. W. Ballard, S. R. Kimmel, B. Ullmann, and T. F. Schilling, "Stages of embryonic development of the zebrafish," Dev. Dyn. 203(3), 253-310 (1995).

19. D. Yelin and Y. Silberberg, "Laser scanning third-harmonicgeneration microscopy in biology," Opt. Express 5(8), 169-175 (1999).

20. W. S. Shan, H. Tanaka, G. R. Phillips, K. Arndt, M. Yoshida, D. R. Colman, and L. Shapiro, "Functional cis-heterodimers of N- and R-cadherins," J. Cell Biol. 148(3), 579-590 (2000).

21. H. Matsunami and M. Takeichi, "Fetal brain subdivisions defined by R- and E-cadherin expressions: Evidence for the role of cadherin activity in region-specific, cell-cell adhesion," Dev. Biol. 172(2), 466-478 (1995).

22. C. H. Kim, E. Ueshima, O. Muraoka, H. Tanaka, S. Y. Yeo, T. L. Huh, and N. Miki, "Zebrafish elav/HuC homologue as a very early neuronal marker," Neurosci. Lett. 216(2), 109-112 (1996).

23. A. B. Gimeno, J. Holzschuh, W. Driever, and E. W. Knapik, "Neural crest survival and differentiation in zebrafish depends on mont blanc/ tfap2a gene function," Development 131(7), 1463-1477 (2004). 